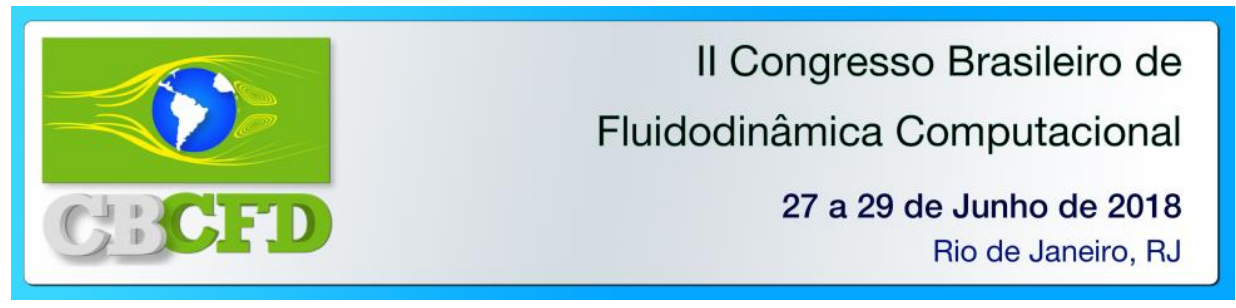

\title{
ANÁLISE DE DISPERSÃO DE GÁS DENSO EM ATMOSFERA ESTÁVEL COM VALIDAÇÃO E VERIFICAÇÃO DOS DADOS
}

\author{
T. F. R. A. RIBEIRO ${ }^{1,2}$, R. A. MEDRONHO ${ }^{1}$ e K. ROPELATO ${ }^{2}$ \\ ${ }^{1}$ Universidade Federal do Rio de Janeiro, Progr. Engenharia de Proc. Químicos e Bioq. \\ ${ }^{2}$ ESSS - Engineering Simulation and Scientific Software \\ E-mail para contato: ribeiro.tfr@gmail.com
}

\begin{abstract}
RESUMO - O presente estudo utilizou os dados experimentais de um cenário do Projeto de Prairie Grass em atmosfera estável para a avaliação da Fluidodinâmica Computacional (CFD) frente a dois métodos analíticos: Método de Pluma Gaussiana (MPG) e Método de Britter-McQuaid (MBQ). O modelo de CFD utilizando entrada logarítmica e modelo de turbulência SST retornou os melhores resultados enquanto que os modelos de CFD alimentados com entrada constante apresentaram baixo desempenho, havendo sempre um modelo analítico de maior robustez. Observou-se que para esta classe de estabilidade atmosférica o MBQ apresentou boa concordância com os dados experimentais, o que não foi visualizado com o MPG devido à natureza de sua formulação.
\end{abstract}

\section{INTRODUÇÃO}

Atualmente, a avaliação da dispersão pode ser realizada através de técnicas experimentais e/ou teóricas. As avaliações experimentais podem ser realizadas em estudos de campo ou em laboratório, utilizando-se modelos em escala reduzida dos cenários que se deseja avaliar. No entanto, normalmente apresentam elevado custo e a desvantagem de fornecer informações sobre as condições atmosféricas apenas para um instante e local, conforme apontado por Pfluck (2010). Os métodos teóricos, compostos basicamente por modelos matemáticos da dispersão de poluentes de natureza numérica ou estatística, podem oferecer, na maioria das vezes, respostas rápidas e de baixo custo para a avaliação destas liberações. Dentre os modelos matemáticos utilizados para este fim, destacam-se o Modelo de Pluma Gaussiana (MPG), o Modelo de Britter-McQuaid (MBQ) e a Fluidodinâmica Computacional (Computational Fluid Dynamics, CFD).

O presente trabalho tem como foco a validação destas três estratégias para a previsão de dispersão de gases frente aos resultados de liberação de $\mathrm{SO}_{2}$ do Projeto de Prairie Grass (Barad, 1958) para um cenário atmosférico estável.

\section{DESCRIÇÃO DO PROBLEMA}

O Projeto Prairie Grass, projetado pelo Air Force Cambridge Research, foi realizado no centro-norte de Nebraska (EUA), no verão de 1956. Pequenas quantidades de dióxido de enxofre ( $\left.\mathrm{SO}_{2}\right)$ puro foram liberadas continuamente por períodos de 10 minutos próximos ao solo para os 68 casos que compuseram o projeto. As medições de dosagem foram feitas em arcos concêntricos localizados a distâncias de 50, 100, 200, 400 e 800 metros na direção do 


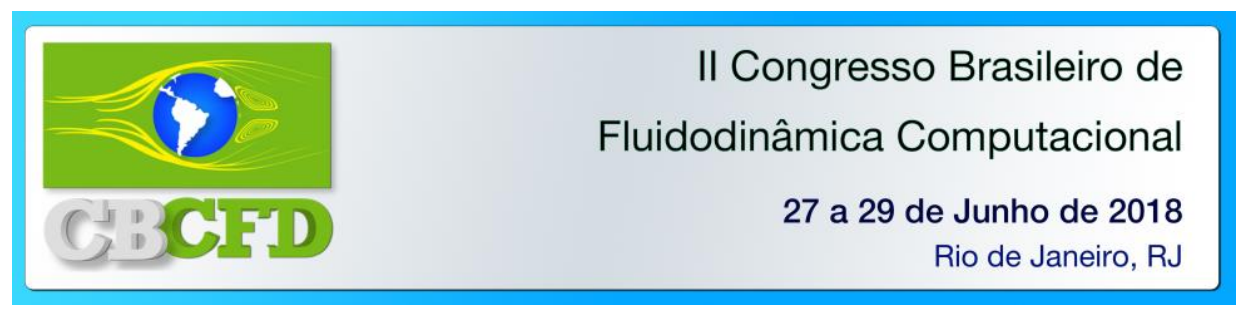

vento. Dentre os cenários disponíveis, foi selecionado um de estratificação estável, pois conforme apontado por Boçon (1988) é esperado que nesta condição os modelos de turbulência isotrópicos não sejam capazes de representar bem a difusão turbulenta. Para avaliação deste comportamento, no presente estudo foram utilizados os modelos $\kappa-\varepsilon$ Scalable e SST.

Como os modelos de CFD geralmente são generalistas, cabendo ao usuário propor quais modelos e condições de contorno empregar, também foram avaliados três diferentes tipos de perfil de vento para a condição de entrada: perfil constante, perfil logarítmico e perfil logarítmico baseado na Teoria de Similaridade de Monin-Obukhov (TSMO). Enquanto a primeira condição não leva em consideração, na sua formulação, a natureza da estabilidade atmosférica, as outras duas tratam essa informação através de diferentes abordagens: o perfil logarítmico utiliza um expoente que correlaciona a classe de estabilidade atmosférica de Pasquill com a natureza do terreno, enquanto que o perfil logarítmico baseado na TSMO utiliza o comprimento de Monin-Obukhov (L) que, para cenários estáveis, encontra-se na faixa $10<L<10^{5}$. A TSMO também prevê perfis para a energia cinética turbulenta $(\kappa)$ e taxa de dissipação viscosa $(\varepsilon)$. Assim, quando utilizado esse perfil juntamente com o modelo $\kappa-\varepsilon$, as variáveis turbulentas foram inicializadas de forma distinta para respeitar a TSMO.

Quando o MPG foi utilizado, foram selecionadas 2 alturas de vento diferentes (1,0 m e $1,5 \mathrm{~m}$ ) para que fosse observada a sensibilidade do modelo a este parâmetro.

As Tabelas 1 a 3 apresentam os dados do cenário de Projeto de Prairie Grass, as definições empregadas na simulação de CFD e as condições de contorno aplicadas no domínio computacional, respectivamente:

Tabela 1 - Condições do Cenário Estável

\begin{tabular}{cccccccc}
\hline $\mathbf{Q}(\mathbf{g} / \mathbf{s})$ & $\mathbf{H s}(\mathbf{m})$ & $\mathbf{Z o}(\mathbf{m})$ & $\boldsymbol{U}_{\mathbf{1 m}}(\mathbf{m} / \mathbf{s})$ & $\mathbf{u}_{*}(\mathbf{m} / \mathbf{s})$ & $\mathbf{T}(\mathbf{C})$ & $\left(\overline{\mathbf{w}^{\prime} \mathbf{\theta}^{\prime}}\right)_{\mathbf{0}}(\mathbf{W} / \mathbf{m} \mathbf{2})$ & $\mathbf{L}(\mathbf{m})$ \\
\hline 56,50 & 0,46 & 0,006 & 2,87 & 0,22 & 27,3 & $-15,71$ & 61,54
\end{tabular}

Onde Q é a vazão mássica do gás liberado, Hs é a altura em que o dióxido de enxofre foi liberado, Zo é a rugosidade do solo, $U_{1 \mathrm{~m}}$ é a altura do vento medido a 1 metro em relação ao solo, $\mathrm{u}_{*}$ é a velocidade de atrito, $\mathrm{T}$ é a temperatura média do ambiente, $\left(\overline{\mathrm{w}^{\prime} \theta^{\prime}}\right)_{0}$ é o fluxo de calor turbulento na superfície do solo e L é o comprimento de Monin-Obukhov.

Tabela 2 - Definições empregadas na simulação de CFD

\begin{tabular}{cc}
\hline Condição & Definição \\
\hline Regime de Escoamento & Estado Estacionário Isotérmico \\
Natureza do Escoamento & Monofásico Multicomponente \\
Materiais & $\mathrm{N} 2$ (Gás Ideal), O2 (Gás Ideal), SO2 (Gás Ideal) \\
Entrada de traçador & Termo Fonte Pontual de Injeção de Material \\
Área de Vazamento & $0,002 \mathrm{~m}^{2}$ \\
Rugosidade do Solo & $0,006 \mathrm{~m}$ \\
Termo Empuxo & Diferença de Massa Específica \\
Modelo de Turbulêcia & $\kappa-\varepsilon$ Scalable e SST com Production and Dissipation \\
Inicialização & Ar: 77\% N2, 23\% O2 \\
Esquema de Advecção & High Resolution \\
Critério de convergência & RSM Max = E-05 \\
\hline
\end{tabular}


II Congresso Brasileiro de

Fluidodinâmica Computacional

27 a 29 de Junho de 2018

Rio de Janeiro, RJ

Tabela 3 - Condições de contorno aplicadas no domínio computacional de CFD

\begin{tabular}{lll}
\hline Região do Domínio & Tipo & Observações \\
\hline Topo & Parede & Parede com deslizamento \\
Solo & Parede & Parede sem deslizamento e com rugosidade \\
Laterais & Simetria & Simetria \\
Entrada & Entrada de Perfil de Velocidade & Perfil de Velocidade Constante, Logarítmica ou Monin-Obukhov de Ar Puro \\
Saída & Opening & Opening com Pressão Atmosférica \\
Emissão & Termo Fonte & Vazão Mássica e Velocidade de emissão do SO2 puro \\
\hline
\end{tabular}

Para o presente trabalho foi utilizado os seguintes softwares da ANSYS na versão 16.1: ANSYS DesignModeler como o CAD para a geração da geometria, ANSYS Meshing como o gerador de malha, ANSYS CFX para o pré-processamento e solução, e ANSYS CFD-Post como o pós-processador.

\section{RESULTADOS E DISCUSSÃO}

A Figura 1 e as Tabelas 4 e 5 apresentam os resultados das simulações via MPG, MBQ e CFD (após o teste de independência de malha) frente aos resultados experimentais.

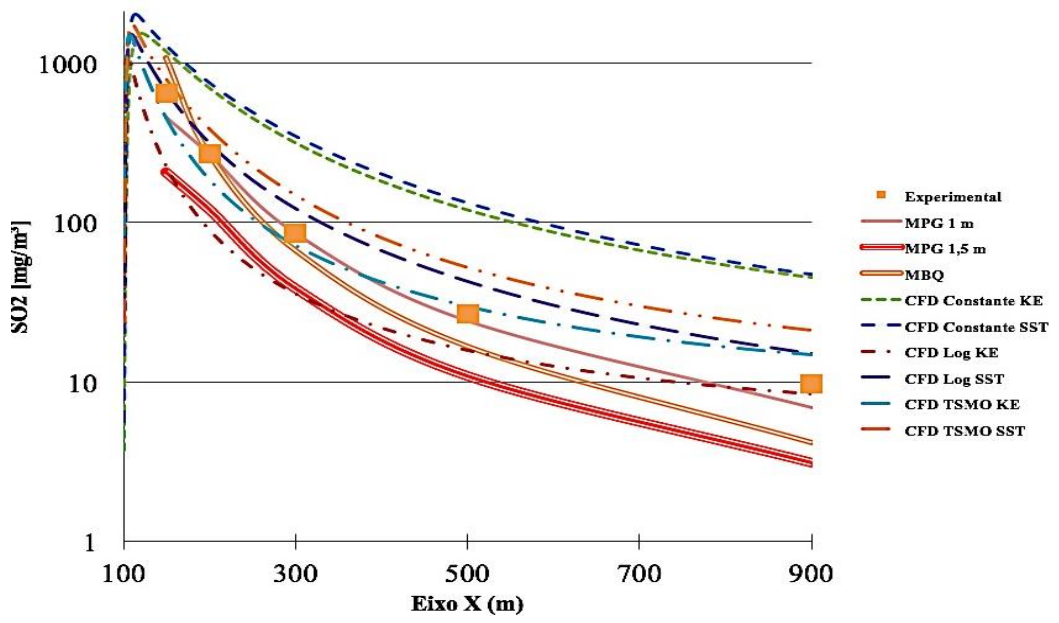

Figura 1 - Perfil de concentração do caso estável.

Tabela 4 - Módulo do erro relativo médio em relação aos resultados do caso estável

\begin{tabular}{|c|c|c|c|c|c|c|c|c|c|}
\hline Distância (m) & MPG $1 \mathrm{~m}$ & MPG 1,5 m & MBQ & CFD Constante KE & CFD Constante SST & CFD Log KE & CFD Log SST & CFD TSMO KE & CFD TSMO SST \\
\hline 150,00 & $64,40 \%$ & $69,11 \%$ & $66,80 \%$ & $80,64 \%$ & $96,88 \%$ & $65,60 \%$ & $1,59 \%$ & $31,50 \%$ & $20,77 \%$ \\
\hline 100,00 & $49,86 \%$ & $56,50 \%$ & $0,02 \%$ & $156,87 \%$ & $177,47 \%$ & $66,74 \%$ & $15,95 \%$ & $31,69 \%$ & $41,96 \%$ \\
\hline 200,00 & $49,93 \%$ & $56,55 \%$ & $21,96 \%$ & $263,92 \%$ & $301,62 \%$ & $58,34 \%$ & $41,43 \%$ & $17,13 \%$ & $71,03 \%$ \\
\hline 400,00 & $54,76 \%$ & $60,75 \%$ & $37,48 \%$ & $343,61 \%$ & $391,67 \%$ & $40,93 \%$ & $59,09 \%$ & $10,60 \%$ & $93,48 \%$ \\
\hline 800,00 & $64,23 \%$ & $68,97 \%$ & $56,83 \%$ & $363,96 \%$ & $384,66 \%$ & $12,83 \%$ & $55,85 \%$ & $52,51 \%$ & $116,30 \%$ \\
\hline Erro Relativo Médio & $56,64 \%$ & $62,37 \%$ & $36,62 \%$ & $241,80 \%$ & $270,46 \%$ & $48,89 \%$ & $34,78 \%$ & $28,68 \%$ & $68,71 \%$ \\
\hline
\end{tabular}

Tabela 5 - Fator de 2 (FAC2) do caso estável

\begin{tabular}{|c|c|c|c|c|c|c|c|c|c|}
\hline Distância (m) & MPG $1 \mathrm{~m}$ & MPG 1,5 m & MBQ & CFD Constante KE & CFD Constante SST & CFD Log KE & CFD Log SST & CFD TSMO KE & CFD TSMO SST \\
\hline 150,00 & 0,36 & 0,31 & 1,67 & 1,81 & 1,97 & 0,34 & 1,02 & 0,68 & 1,21 \\
\hline 200,00 & 0,50 & 0,44 & 1,00 & 2,57 & 2,77 & 0,33 & 1,16 & 0,68 & 1,42 \\
\hline 300,00 & 0,50 & 0,43 & 0,78 & 3,64 & 4,02 & 0,42 & 1,41 & 0,83 & 1,71 \\
\hline 500,00 & 0,45 & 0,39 & 0,63 & 4,44 & 4,92 & 0,59 & 1,59 & 1,11 & 1,93 \\
\hline 900,00 & 0,36 & 0,31 & 0,43 & 4,64 & 4,85 & 0,87 & 1,56 & 1,53 & 2,16 \\
\hline FAC2 & $40,00 \%$ & $0,00 \%$ & $80,00 \%$ & $20,00 \%$ & $20,00 \%$ & $40,00 \%$ & $100,00 \%$ & $100,00 \%$ & $80,00 \%$ \\
\hline
\end{tabular}

Nota-se que as simulações de CFD com perfil de velocidade constante, apesar de conservadores, obtiveram os maiores erros relativos médios e baixo FAC2.

Os cenários do MPG, CFD com condição de entrada logarítmica e modelo $\kappa-\varepsilon$ (CFD Log KE) e CFD com condição de entrada baseada na TSMO e modelo SST (CFD TSMO 


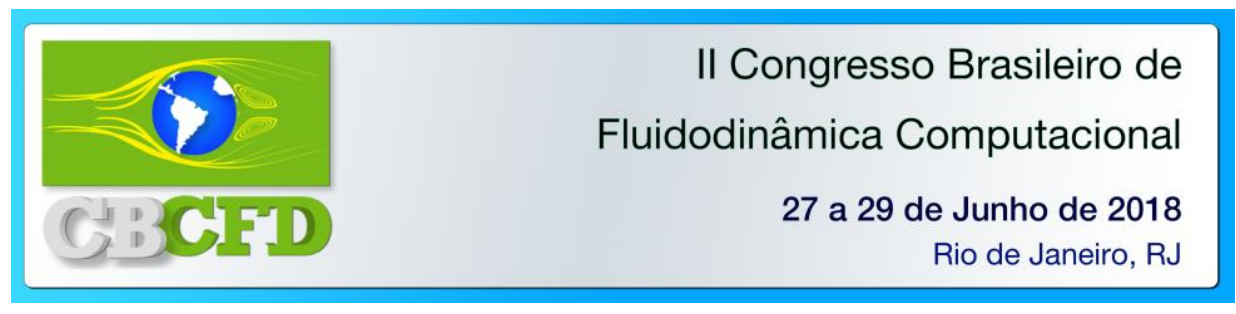

SST) apresentaram resultados intermediários. Observou-se que apesar do aumento de $0,5 \mathrm{~m}$ na altura de medição do vento no MPG elevar ligeiramente o erro relativo, o FAC2 caiu de $40 \%$ para $0 \%$. Isso ocorre, pois quanto mais estável o cenário, maior a variação do vento em relação à altura, tornando este método bastante sensível a esta variável. Outro ponto relevante é que em ambos os casos o MPG subestimou a concentração em todos os pontos.

O MBQ apresentou resultados melhores que o MPG em relação ao erro relativo médio e FAC2. Contudo, as concentrações foram subestimadas nos últimos três arcos de medição, o que torna este método conservador apenas para regiões próximas da liberação.

As estratégias empregando-se CFD com condição de entrada logarítmica e modelo SST (CFD Log SST) e condição logarítmica baseada na TSMO e modelo $\kappa-\varepsilon$ (CFD TSMO KE) apresentaram boa concordância em relação ao erro relativo médio e FAC2 de $100 \%$. No entanto, apesar do CFD TSMO KE apresentar o menor erro entre os dois, provavelmente pela inicialização das variáveis turbulentas, essa estratégia subestima os três primeiros arcos de medição, tornando-a menos conservadora para análise de dispersão que o CFD Log SST.

\section{CONCLUSÃO}

Conclui-se então que para o cenário estável o modelo CFD Log SST aparenta o melhor compromisso entre erro relativo médio, erro relativo ponto a ponto, FAC2 e conservadorismo, por superestimar a concentração de $\mathrm{SO}_{2}$ em todos os arcos de medição. Caso este último critério não estivesse sendo avaliado, o modelo CFD TSMO $\kappa-\varepsilon$ Scalable seria escolhido como a melhor opção, apresentado o erro relativo médio mais baixo dentre todos os cenários provavelmente pela inicialização utilizada das variáveis turbulentas.

Observou-se que no caso de dispersão de um gás denso próximo ao solo em atmosfera estável é preferível utilizar um método analítico a uma simulação via CFD utilizando entrada constante. Isso ocorre, pois este método não leva em conta a estabilidade atmosférica e não representa bem o perfil de velocidade próximo ao solo. Em condições a alturas mais altas, em atmosfera neutra ou instável e com gases leves, este cenário talvez possa ser diferente.

O MPG deve ser utilizado com muita cautela em cenários estáveis em gases densos, pois além de não incluir informação sobre o empuxo em sua formulação, o mesmo é altamente sensível à medição da altura do vento. Em atmosferas estáveis, onde existe uma maior variação do perfil com a altura do que nos casos instáveis, essa medição pode levar a uma má interpretação dos dados disponíveis.

\section{REFERÊNCIAS}

BOÇON, F.T. Modelagem Matemática do Escoamento e da Dispersão de Poluentes na Microescala Atmosférica, Tese de Doutorado, Universidade Federal de Santa Catarina, Florianópolis, 1998.

PFLUCK, C. E. F. Simulação Fluidodinâmica da Dispersão de Poluentes na Atmosfera, Dissertação de Mestrado, Universidade Federal do Rio Grande do Sul, 2010.

BARAD, M.L. 1958. Project Prairie Grass: A Field program in diffusion, Geophys. Res. Paper N 59 (II) TR-58-235 (II), Air Force Cambridge Research Centre, USA 